\title{
Physical Basis for Membrane-Charge Selectivity of Cationic Antimicrobial Peptides
}

\author{
Sattar Taheri-Araghi and Bae-Yeun Ha \\ Department of Physics and Astronomy, University of Waterloo, Waterloo, Ontario N2L 3G1, Canada
}

(Received 15 June 2006; published 16 April 2007)

\begin{abstract}
Antimicrobial peptides are known to selectively disrupt (highly charged) microbial membranes by asymmetrical incorporation into the outer layers. We present a physical basis for membrane-charge selectivity of cationic antimicrobial peptides. In particular, we provide a clear picture of how peptidecharge $Q$ influences the asymmetrical insertion-one salient feature is the existence of an optimal peptide charge, at which selective insertion is optimized. Our results suggest that large $Q$ is required for antimicrobial selectivity, consistent with experiments.
\end{abstract}

PACS numbers: $87.15 . \mathrm{Kg}, 87.16 . \mathrm{Dg}$

Antimicrobial peptides are self-defense molecules of multicellular organisms that fend off invading microbes (e.g., bacteria and fungi) [1]. The peptides "selectively" disrupt microbial lipid membranes without harming multicellular, host cell membranes [1-3]. How can this be accomplished? Along this line, cationic peptides such as magainin II are of particular interest - they differentiate between target (e.g., bacterial) membranes and host (i.e., animals and plants) cell membranes primarily through their interaction with anionic lipids rather than receptormediated recognition processes [1-3]: The outer (or outmost) layer of bacterial membranes contains a large fraction of anionic lipids, while the outer layer of host cell membranes mainly consists of lipids with electrically neutral headgroups (see Ref. [1], and references therein). This allows the peptide to selectively bind to a bacterial membrane (in a peptide-charge and -concentration dependent way). The binding is initially driven by the electrostatic attraction between the peptide and anionic lipids, and then by the hydrophobic attraction between the peptide and lipids. Owing to their amphiphilic nature, these peptides can also insert into the outer layer-note the binding is initially "asymmetrical." The asymmetrical insertion will thus create a mechanical stress on the bilayer, priming the bilayer for membrane rupture [4], likely through pore formation [5-7].

Despite the wealth of experimental data and despite its practical significance in biomedical technology (e.g., the rational design of antibiotics), a comprehensive, quantitative description of the electric-hydrophobic binding is still illusive: Even though many experiments highlight unambiguously the role played by electrostatic interactions (see, for example, Refs. [2,3]), theoretical understanding of selective binding remains poor. The main difficulty arises from the subtle but important interplay between lipid rearrangements and peptide binding, which have to be determined simultaneously.

In this Letter, we seek to present a theoretical model for describing the association of cationic peptides with a lipid membrane - in an effort to offer a quantitative basis for some of major advances in experimental counterparts.
Owing to the complexity of the problem, however, one has to invoke simplification, so as to focus on important degrees of freedom. The model membrane we employ is a pure-lipid bilayer, possibly containing anionic lipids, immersed in electrolyte solutions. Peptides are modeled as a thin circular disk carrying a charge $Q$; molecular details such as their hydrophobicity will be subsumed into a few parameters. Recent work on magainin II analogs indicates that $Q$ and peptide's hydrophobicity can be modified almost independently [8]. Similarly we consider them as independent parameters. This simplification allows us to identify important parameters that affect selectivity and to understand the molecular origin of selectivity.

A few novel features emerge from our consideration. First, we find that there exists an optimal peptide charge, $Q^{*}$ ( $\approx 5-6$ for magainin II analogues), at which selective insertion of the peptide is optimized. Here, selectivity refers to the ability of peptides to preferentially penetrate into a highly charged outer layer. This naturally arises from the fact that peptide's binding affinity for a highly charged surface can be maximized at a certain value of $Q$, but it decreases monotonically with $Q$ for a neutral surface. Also, our results not only show how $Q$ and peptide's hydrophobicity are intertwined in determining the hydrophobic insertion but also are consistent with high- $Q$ requirements for antimicrobial selectivity [8-10]. They indicate that, to optimize the selectivity, larger $Q$ is needed for higher hydrophobicity. Finally, we find that peptide insertion is enhanced by surface-adsorbed peptides (i.e., those adsorbed electrically onto the membrane surface), which tend to catalyze the insertion by reducing the optimal headgroup area of lipids in their binding layer.

To describe the electric-hydrophobic binding, we first classify peptides into three subclasses in chemical equilibrium: "free" (those in bulk), "surface-adsorbed" (those adsorbed electrically at the water-headgroup interface), and "inserted" (those inserted in the lipids, more precisely at the interface between the headgroups and the tails [5-7], thus interacting with the lipids both hydrophobically and electrically). By balancing the chemical potentials of three species, one can find the equilibrium planar concentrations 
of surface-adsorbed and inserted peptides, denoted by $\sigma_{S}$ and $\sigma_{I}$, respectively.

The energy gain is different for different binding modes. Each inserted peptide compresses the surrounding lipids $[5-7,11]$ through steric repulsion, costing an elastic energy. Note that this effect should compete with opposing effects: Bound peptides (those surface adsorbed or inserted) can reduce the relaxed area of the outer layer [12] — the attraction of the anionic lipids to bound peptides results in a partial compression of the lipids. Let $\delta a_{i}(i=$ " $S$ " or " $I$ ") be the relaxed-area change per peptide. The fractional area change of the outer layer is then $\Delta A / A \approx$ $\sigma_{I} a_{\|}+\sigma_{I} \delta a_{I}+\sigma_{S} \delta a_{S}$, where $A$ is the area of the outer layer and $a_{\|}$is the area of an inserted peptide, assumed to be oriented parallel with the bilayer $[5,11]$. The resulting elastic free energy per area is then $\mathcal{F}_{\mathrm{el}} \approx \frac{1}{2} K_{A}\left(\sigma_{I} a_{\|}+\right.$ $\left.\sigma_{I} \delta a_{I}+\sigma_{S} \delta a_{S}\right)^{2}$, where $K_{A}$ is the area-stretch modulus [13]. The effect of $\delta a_{i}(<0)$ is to reduce the elastic energy cost for insertion. In this sense, surface adsorption can promote insertion, as will be detailed below.

As for the electric binding of bound peptides, we will essentially follow up on the approaches introduced in Refs. [14,15] with some modifications. The peptides are modeled as a uniformly charged hard disc (of area $a_{\|}$and charge $Q$ ). The membrane is assumed to be flat and aligned in the $(x, y)$ plane; the outer layer is at $z=0$ and the inner one is at $z=-d$. Anionic lipids can readjust their position and thus their spatial distributions in response to other ions, especially bound peptides - the resulting approach will permit a systematic treatment of charge correlations and lipid demixing or rearrangement. To allow these degrees of freedom, we introduce a spatially varying lipid composition (or fraction) of anionic lipids at $z=0, \alpha(x, y)$. If $\bar{\alpha}$ is the average fraction, then $\bar{\alpha}=\int_{A} \alpha(x, y) d x d y / \int d x d y$. In this electrostatic calculation, bound peptides (whether surfaced adsorbed or inserted) are assumed to be in the plane of the outer layer at $z=0$, forming a two-dimensional hexagonal lattice due to their mutual repulsion; each peptide defines a "Wigner-Seitz cell" (WSC) of radius $R_{\mathrm{Ws}}$. The Poisson-Boltzmann (PB) approach will be used to calculate the electrostatic interaction between anionic lipids and a peptide in a WSC [thus to find $\alpha(x, y)$ and $\sigma_{i}$ ]; the boundary condition at $r=R_{\mathrm{WS}}$ approximately captures the electrostatic repulsion between peptides, as influenced by the surrounding anionic lipids and salts [14]. The salts are assumed to be monovalent, even though in principle multivalent salts can be included.

Let $\Phi(\mathbf{r})$ be the electrostatic potential at $\mathbf{r}$. It proves useful to introduce the reduced potential $\Psi=e \Phi / k_{B} T$, where $e$ is the electronic charge, $k_{B}$ the Boltzmann constant, and $T$ the temperature. In the aqueous phase (i.e., $z>0$ or $z<-d), \Psi$ satisfies the PB equation: $\nabla^{2} \Psi(\mathbf{r})=$ $\kappa^{2} \sinh \Psi(\mathbf{r})$, where $\kappa^{-1}$ is the Debye screening length, a length beyond which the electrostatic interaction is exponentially screened.

To relate $\alpha(x, y)$ to $\Psi_{0}(x, y) \equiv \Psi(x, y, z=0)$, we construct a free energy functional for the peptide binding layer, i.e., $z=0$. If $a_{l}$ is the lipid headgroup area, the local surface charge density is simply $\sigma_{l}(x, y)=-\left(e / a_{l}\right) \times$ $\alpha(x, y)$. The free energy functional within each WSC at the PB level is given by

$$
\begin{aligned}
\frac{F_{\mathrm{PB}}}{k_{B} T}= & \frac{1}{a_{l}} \iint\left[\alpha \ln \frac{\alpha}{\bar{\alpha}}+(1-\alpha) \ln \frac{1-\alpha}{1-\bar{\alpha}}\right] d x d y \\
& -\frac{e}{a_{l}} \iint \alpha \Psi_{0} d x d y+\frac{\lambda}{a_{l}} \iint(\alpha-\bar{\alpha}) d x d y,
\end{aligned}
$$

where the integrals are over the area of a WSC. The first term describes the entropic penalty for the modulation of anionic-lipid distributions. The second term accounts for the electrostatic energy of anionic lipids. The last term ensures the charge conservation $\int \alpha d x d y=\pi \bar{\alpha} R_{\mathrm{WS}}^{2}$. The Lagrange multiplier $\lambda$ should be adjusted so as to satisfy charge conservation.

Minimization of $F_{\mathrm{PB}}$ with respect to $\alpha(x, y)$ leads to the spatial distribution of anionic lipids: $\alpha=e^{\Delta \Psi_{0}} /\left[\frac{1-\bar{\alpha}}{\bar{\alpha}}+\right.$ $\left.e^{\Delta \Psi_{0}}\right]$, where $\Delta \Psi_{0}=\Psi_{0}-\lambda$. The charge conservation requires $\int d x d y e^{\Delta \Psi_{0}} /\left[\frac{1-\bar{\alpha}}{\bar{\alpha}}+e^{\Delta \Psi_{0}}\right]=\pi R_{\mathrm{WS}}^{2} \bar{\alpha}$.

The normal component of an electric field across a charged surface is discontinuous. The boundary condition at $z=0$ is $-e\left(a_{l} / 4 \pi \ell_{B}\right)(\partial \Psi / \partial z)_{z=0}=\left(-e \alpha / a_{l}\right) \delta_{i S}+$ $Q / a_{\|}$for $r<r_{\|}$or $-e \alpha / a_{l}$ for $r>r_{\|}$, where $i=S$ or $I$, $r_{\|}$is the peptide radius, and $\ell_{B}=e^{2} / \epsilon_{w} k_{B} T$ the Bjerrum length with $\epsilon_{w}$ the dielectric constant of water [16]. (This boundary condition is different for the two binding modes.) Besides, $\Psi$ should satisfy an additional boundary condition at $r=R_{\mathrm{WS}}$. If only one kind of binding is considered, we impose $(\partial \Psi / \partial r)_{r=R_{\mathrm{WS}}}=0$ [14]. Such a simple relation, which solely arises from symmetry, does not exist for the current problem, since different binding modes present different boundary conditions at $r=R_{\mathrm{WS}}$. Nevertheless, we use $(\partial \Psi / \partial r)_{r=R_{\mathrm{WS}}}=0$ for a bound peptide at $r=0$, independently of the binding modes of its surrounding peptides. The validity of this can be checked a posteriori [17]. We will then solve the PB equation, subject to all the boundary conditions, with nonuniform distributions of anionic lipids around a bound peptide [18].

In this Letter, we determine the relaxed area of the outer layer and electrostatics simultaneously. To this end, it suffices to focus on one WSC, which can be pictured as a charged elastic sheet of radius $R_{\mathrm{Ws}}$. Let $U(r)$ be the displacement of the sheet at $r$ and $\Pi(r)$ the $r$ component of the electrostatic force per area at $r$ (the normal component is irrelevant). By balancing elastic and electrostatic forces at $r$, one can show that $r^{2} d^{2} U(r) / d r^{2}+r d U(r) / d r-$ $U(r)=r^{2} \Pi(r) / K_{A}$. This can be solved with appropriate boundary conditions, details of which will be reported elsewhere. The resulting relaxed-area change per WSC (or per peptide) is $\delta a_{i}=\pi\left[U^{2}\left(R_{\mathrm{WS}}\right)+2 R_{\mathrm{WS}} U\left(R_{\mathrm{WS}}\right)\right]$, which influences the elastic free energy (per area) $\mathcal{F}_{\text {el }}$.

The total free energy per area is given by

$$
\begin{aligned}
\mathcal{F}= & \mathcal{F}_{\mathrm{el}}+\sigma_{S} F_{S}+\sigma_{I} F_{I}+\sigma_{I} \varepsilon_{I} \\
& +k_{B} T\left[\sigma_{S}\left(\ln \sigma_{S} a_{\|}-1\right)+\sigma_{I}\left(\ln \sigma_{I} a_{\|}-1\right)\right] .
\end{aligned}
$$


Here $F_{i}(i=S$ or $I)$ is the electrostatic free energy of one WSC - this is simply the sum of $F_{\mathrm{PB}}$ and the contribution of salts with reference to the corresponding unperturbed case: a system of a free peptide in bulk and an unperturbed bilayer. On the other hand, $\varepsilon_{I}$ is a phenomenological parameter that accounts for the hydrophobic free energy gain of each inserted peptide. Measurements using isothermal calorimetry [19] indicates that the $\varepsilon_{I}$ for the binding of magainin and mellittin is in the range $-7.0 \mathrm{kcal} / \mathrm{mol} \approx$ $-12 k_{B} T$ per peptide (throughout this Letter $T$ is chosen to be room temperature, $T=300 \mathrm{~K}$ ). The last term in Eq. (2) describes the entropy of bound peptides, which is accurate for $\sigma_{i} \ll 1 / a_{\|}(i=S$ or $I)$, as is often the case (see Fig. 1).

In equilibrium, the chemical potentials of surfaceadsorbed and inserted peptides are equal to that of free peptides, $\mu_{\text {free }}: \partial \mathcal{F} / \partial \sigma_{I}=\partial \mathcal{F} / \partial \sigma_{S}=\mu_{\text {free }}$; in our model, $\mu_{\text {free }} \approx \ln \left(n_{\text {bulk }} v\right)$, where $n_{\text {bulk }}$ is the bulk peptide concentration and $v$ is the volume of peptides. Our numerical scheme is equivalent to minimizing the free energy with respect to $R_{\mathrm{WS}}$ and the fraction of inserted peptides, $f_{I}=\sigma_{I} /\left(\sigma_{S}+\sigma_{I}\right)$.

Some experimental studies [5,6] indicate that surface activity of antimicrobial peptides (prior to pore formation) is well quantified by a single parameter: the molar ratio of inserted peptides to lipids, denoted by $P_{I} / L=\sigma_{I} / \sigma_{l}=$ $\sigma_{I} a_{l}$, where $\sigma_{l}=1 / a_{l}$. More generally, Ref. [20] shows how a membrane can be permeabilized by asymmetrical incorporation of molecules. In Fig. 1, we have plotted a few relevant quantities as a function of $Q$. The top graph is $P_{I} / L$ vs $Q$ for two different choices of $\bar{\alpha}: \bar{\alpha}_{>}=0.30$ (thick lines with unfilled squares) and $\bar{\alpha}_{<}=0.05$ (thin lines with filled squares) - the former represents the outer layer of a typical bacterial membrane. Additionally, we have chosen $T=300 \mathrm{~K}, a_{\|}=3.1 \mathrm{~nm}^{2}, a_{l}=$ $0.8 \mathrm{~nm}^{2}, \epsilon_{w}=80$ (see endnote [16]), $\kappa^{-1}=10 \AA, K_{A}=$ $0.06 k_{B} T / \AA^{2}$ [21], and $n_{\text {bulk }}=5 \mu \mathrm{M}$, a typical concentration required for the rupture of bacterial membranes. On the other hand, we have used three choices of $\varepsilon_{I}: \varepsilon_{I}=$ $-10,-12$, and -16 in units of $k_{B} T(T=300 \mathrm{~K})$.

Perhaps, the most salient feature is the existence of an optimal peptide charge $Q^{*}$, at which selectivity is optimized. Here selectivity is measured by $\Delta\left(P_{I} / L\right)=$ $\left(P_{I} / L\right)_{\bar{\alpha}_{>}}-\left(P_{I} / L\right)_{\bar{\alpha}_{<}}[22] ; \Delta\left(P_{I} / L\right)$ for $\varepsilon_{I}=-12 k_{B} T$ is described by the dotted line. This can be understood in parallel with the nonmonotonic behavior of $\left(P_{I} / L\right)_{\alpha_{>}}$as a function of $Q$. For $\varepsilon_{I}=-12 k_{B} T$, a typical value for magainin II, $\left(P_{I} / L\right)_{\alpha_{>}}$is maximized at $Q \approx 4$. (It is gratifying to note that this charge characterizes magainin II.) Crudely speaking, the peptide-peptide repulsion hinders peptide binding more efficiently for large $Q$ (also see below for relevant discussion). On the other hand, for $\bar{\alpha}=$ $\bar{\alpha}_{>}$, this unfavorable repulsion is partly compensated by the enhanced electrostatic attraction between peptides and lipids. It is conceivable that the balance of the two is responsible for the nonmonotonic dependence. This naturally gives rise to an optimal charge $Q^{*}$, at which the

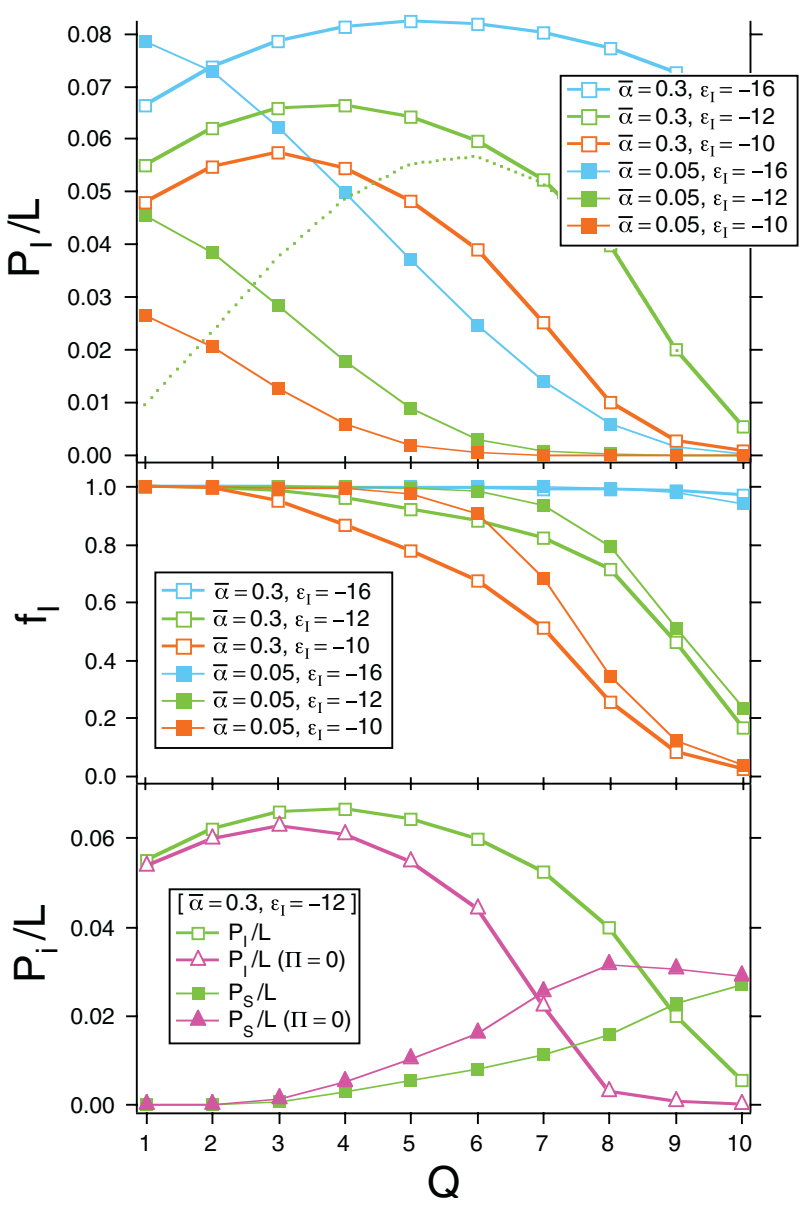

FIG. 1 (color). Graphs of $P_{I} / L$ (molar ratio of inserted peptides to lipids), $f_{I}$ (fraction of inserted peptides), and $P_{i} / L$ (molar ratio of bound peptides to lipids) with $i=I$ (inserted) or $S$ (surface adsorbed), as a function of $Q$ for different choices of $\varepsilon_{I}$ and $\bar{\alpha}$ (see the legend). We have chosen $T=300 \mathrm{~K}, \epsilon_{w}=$ 80 (see also [16]), $n_{\text {bulk }}=5 \mu \mathrm{M}, \kappa^{-1}=10 \AA, a_{\|}=3.1 \mathrm{~nm}^{2}$, $a_{l}=0.8 \mathrm{~nm}^{2}$, and $K_{A}=0.06 k_{B} T / \AA^{2}$. The top figure indicates that there exists an optimal charge, $Q^{*}$, at which $\Delta\left(P_{I} / L\right)=$ $\left(P_{I} / L\right)_{\bar{\alpha}=0.3}-\left(P_{I} / L\right)_{\bar{\alpha}=0.05}$ is maximized; see the dotted line for $\varepsilon_{I}=-12$ (in units of $k_{B} T$ ). Note $Q^{*}$ is larger for a larger $\varepsilon_{I}$. As $Q$ increases, $f_{I}$ decreases, implying that bound peptides preferentially reside on the membrane surface for large $Q$ (the middle graph), as also indicated in the bottom graph for the fraction $\bar{\alpha}=0.3$ - as $Q$ increases, $P_{S} / L$ increases, while $P_{I} / L$ decreases beyond $Q \approx 4$. The curves with triangles in the bottom plot were constructed with $\Pi$ (the electrostatic lateral pressure) set to zero, showing how bound peptides, especially surfaced-adsorbed ones, catalyze insertion. (See the text for details.)

selectivity is optimized (in an $\varepsilon_{I}$-dependent way). For $\varepsilon_{I}=$ $-12 k_{B} T$, which typifies magainin II, $Q^{*} \approx 5-6$ (see the dotted line). In an experimental study [8], the charge of magainin II analogs was modulated between 3 and 7 with other parameters conserved. Antimicrobial selectivity was then studied by dye release (see Ref. [8]) - selectivity in this context refers to the ability to selectively rupture microbial membranes. This led to the observation that antimicrobial selectivity is optimized at $Q \approx 5$. Even 
though the optimal charge for $\Delta\left(P_{I} / L\right)$ can be different from that for antimicrobial selectivity, the agreement of this with ours is not accidental, since antimicrobial activity is related to $\left(P_{I} / L\right)_{\alpha_{>}}$.

Obviously, $P_{I} / L$ is larger for a larger $\varepsilon_{I}$. This implies that antimicrobial activity can be enhanced by increasing $\varepsilon_{I}$. Recall that activity of the peptides prior to membrane rupture is controlled by $\left(P_{I} / L\right)_{\alpha_{>}}[5,6]$. For a large $\varepsilon_{I}$, however, high valences are required to reduce $\left(P_{I} / L\right)_{\alpha}$ and thus hemolytic activity (activity against weakly or neutral membranes) so as to retain high selectivity, as evidenced in the top figure. This finding is indeed in accord with the experimental observation of high-valence requirements for antimicrobial selectivity [8-10]. An experimental study [9], in which the hydrophobicity of magainin II analogs was modulated with other parameters fixed (e.g., $Q=4$ ), suggests that increasing hydrophobicity results in reduced selectivity. This compares favorably with the results in the top figure - the selectivity for $Q=4$ decreases as $\varepsilon_{I}$ changes from $-12 k_{B} T$ to $-16 k_{B} T$.

Interestingly, bound peptides tend to reside on the membrane surface for a large $Q$, as the graph of $f_{I}$ vs $Q$ indicates. This is understandable, since the peptide charge is more efficiently shielded by anionic lipids when they are surface adsorbed. This is partly responsible for the rapid decrease of $P_{I} / L$ with increasing $Q$ for large $Q$, as is particular the case for $\varepsilon_{I}=-10$ and -12 (in units of $\left.k_{B} T\right)$. As a result, charged-membrane rupture occurs in a narrower range of $Q$ than it would otherwise. In other words, this effect enhances peptide-charge selectivity for membrane rupture.

We have also studied the effect of bound peptides on insertion. In the bottom figure $\left(\bar{\alpha}=0.3\right.$ and $\varepsilon_{I}=$ $\left.-12 k_{B} T\right)$, the curves with triangles, obtained with $\Pi$ set to zero, are compared with those with this effect incorporated. Interestingly, this effect adds to selectivity, by lowering the energy barrier to insertion; like simple multivalent ions (e.g., $\mathrm{Ca}^{2+}$ ) [12], they tend to shrink the optimal headgroup area of lipids in the outer layer, catalyzing insertion. For $Q \lesssim 8$, this cooperative feat, as described by the difference in $P_{I} / L$ between the two cases (squares and triangles), is more effective for a large $Q$; beyond $Q \approx 8$, bound peptides mainly reside on the membrane surface so as to diminish the peptide-peptide repulsion as discussed above. The results also indicate that surfaced-adsorbed peptides enhance insertion more efficiently: The difference in $P_{I} / L$ between the two cases is mainly due to surface-adsorbed ones (up to $Q \approx 8$ ).

In conclusion, we have presented a quantitative basis for membrane-charge selectivity of cationic antimicrobial peptides. The peptides ultimately target lipid membranes with a broad spectrum of antimicrobial activity against many species: (Gram-positive and -negative) bacteria, fungi, viruses, and some tumor cells. A number of experimental studies [8-10] unambiguously suggest that peptide charge is a dominating factor in determining membrane selectivity. The theoretical approach developed here offers a molecular origin of the selectivity.

We acknowledge financial support from NSERC (Canada). We are grateful to H. W. Huang, A. Tossi, and S. Jun for useful discussions.

[1] M. Zasloff, Nature (London) 415, 389 (2002).

[2] M. R. Wenk and J. Seelig, Biochemistry 37, 3909 (1998).

[3] K. Matsuzaki, Biochim. Biophys. Acta 1462, 1 (1999).

[4] The peptide ruptures the bacterial membrane at micromolar concentrations [1]; much higher, milimolar concentrations are, however, needed to rupture membranes whose outer layers are electrically neutral [3].

[5] F.-Y. Chen et al., Biophys. J. 84, 3751 (2003).

[6] H. Huang et al., Phys. Rev. Lett. 92, 198304 (2004).

[7] M.-T. Lee et al., Biochemistry 43, 3590 (2004).

[8] M. Dathe et al., FEBS Lett. 501, 146 (2001).

[9] T. Wieprecht et al., Biochemistry 36, 6124 (1997).

[10] K. Matsuzaki et al., Biochemistry 36, 2104 (1997).

[11] H. W. Huang, J. Phys. II 5, 1427 (1995).

[12] Y. Li and B.-Y. Ha, Europhys. Lett. 70, 411 (2005).

[13] In fact, $K_{A}$ is influenced by peptide binding. This effect is, however, a small correction and will be ignored-see Ref. [12] for similar issues. Asymmetrical peptide binding can also induce a spontaneous curvature $C_{0}$, which will change the energetics of peptide insertion. We have checked that the corresponding free energy cost (per area) is much smaller than $\mathcal{F}_{\text {el }}$ considered in this Letter.

[14] S. May, D. Harries, and A. Ben-Shaul, Biophys. J. 79, 1747 (2000).

[15] D. Murray et al., Biophys. J. 77, 3176 (1999).

[16] It has been known [14] that two layers of a lipid bilayer are electrically decoupled for $\kappa d \epsilon_{w} / \epsilon_{l} \gg 1$, where $\epsilon_{w}$ and $\epsilon_{l}$ are the dielectric constants of water and lipids, respectively, given by $\epsilon_{w}=80$ and $\epsilon_{l}=2$. This is equivalent to ignoring a term containing $\epsilon_{l}(\partial \Psi / \partial z)_{z=0^{-}}$in the boundary condition at $z=0$. For typical values of $\kappa^{-1}(\sim 10 \AA)$ and $d(\sim 40 \AA)$, this inequality can easily be satisfied. This allows us to focus on the peptide binding layer, i.e., the outer layer, in our electrostatic calculations. For a surfaceadsorbed peptide, our boundary condition is, in fact, the same as the one used in Ref. [14].

[17] The error caused by this simplification is minimal if $f_{I} \simeq 0$ or $f_{I} \simeq 1$. For an intermediate range of $f_{I}$, we have checked that the error/WSC is much smaller than our estimate of $F_{\mathrm{PB}}$.

[18] In our electrostatic calculations, we assume that peptide binding would not change the "physical" area of the outer layer, $A$. Ideally, one can consider simultaneously peptide binding and membrane deformations it causes. We believe that this will lead to higher order effects.

[19] T. Wieprecht, M. Beyermann, and J. Seelig, Biochemistry 38, 10377 (1999).

[20] H. Heerklotz, Biophys. J. 81, 184 (2001).

[21] We have chosen this value as an energy cost per area for stretching the outer layer at the headgroup-lipid interface.

[22] This is such that the peptide is antimicrobial-active for $\bar{\alpha}=\bar{\alpha}_{>}$, but not for $\bar{\alpha}=\bar{\alpha}_{<}$. 